\title{
Efficacy and Tolerability of Exenatide Once Weekly Over 6 Years in Patients with Type 2 Diabetes: An Uncontrolled Open-Label Extension of the DURATION-1 Study
}

\author{
Robert R. Henry, MD, Eric J. Klein, MD, Jenny Han, $M S^{3}$ and Nayyar lqbal, MD ${ }^{4}$
}

\begin{abstract}
Background: Long-term treatment is necessary to slow the progression of type 2 diabetes (T2D). Here, we examined the safety and efficacy of 6 years of treatment with exenatide once weekly (QW) among patients with T2D in the DURATION-1 trial.

Methods: The study enrolled patients aged $\geq 16$ years with T2D treated primarily with metformin and sulfonylureas. Following 30 weeks of randomized treatment with exenatide QW $2 \mathrm{mg}$ or exenatide twice daily $10 \mu \mathrm{g}$, patients entered an uncontrolled, open-label, open-ended study phase in which all patients received exenatide QW $2 \mathrm{mg}$. Restrictions on concomitant medication use were eased over time.

Results: Of the original 295 patients in the intent-to-treat population, $136(46 \%)$ completed 6 years of treatment. Six-year completers had sustained significant improvements from baseline in glycated hemoglobin (HbAlc; least-squares mean [LSM] change, $-1.6 \%$ ), with $46.3 \%$ achieving $\mathrm{HbA} 1 \mathrm{c}<7.0 \%, 33.1 \%$ achieving $\mathrm{HbA} 1 \mathrm{c}$ $\leq 6.5 \%$, and significant improvements from baseline in fasting plasma glucose $(-28 \mathrm{mg} / \mathrm{dL})$ and body weight $(-4.2 \mathrm{~kg})$ at 6 years. The 78 completers who added no glucose-lowering medications had numerically greater body weight reductions than the overall cohort (6-year LSM change, $-6.1 \mathrm{~kg}$ ) with more stability over time. No unexpected adverse events were observed during 1202.4 patient-years of exposure. Most minor hypoglycemia events occurred with concomitant sulfonylurea use.

Conclusions: Exenatide QW was associated with clinically significant, sustained improvements in glycemic control and weight in patients who continued therapy for up to 6 years, without unexpected safety findings. ClinicalTrials.gov registration: NCT00308139.
\end{abstract}

\section{Introduction}

$\mathbf{T}$ YPE 2 DIABETES (T2D) is a progressive disease, in which an ongoing decline in $\beta$-cell function is associated with glycemic deterioration over time. ${ }^{1}$ Treatment guidelines generally recommend a sequential approach to treatment of T2D, with stepwise escalation of treatment if the glycated hemoglobin (HbA1c) target is not met after $\sim 3$ months. ${ }^{2}$ Due to the need for lifetime pharmacotherapy, patients may remain on therapies for long periods of time without data to inform their likely course. Thus, long-term data on the efficacy and safety of glucose-lowering therapies are important.
However, long-term clinical studies are not often conducted due to the challenges of retaining patients in a trial over time and the considerable effort required from patients, coordinators, and investigators to continue the study.

A microsphere-based extended-release formulation of the glucagon-like peptide-1 receptor agonist (GLP-1RA) exenatide, which is administered subcutaneously once weekly (QW), was initially studied in the Diabetes Therapy Utilization: Researching Changes in A1C, Weight, and Other Factors Through Intervention With Exenatide Once Weekly (DURATION-1) trial. This study, conducted in patients with T2D treated with lifestyle modification or oral

\footnotetext{
${ }^{1}$ Center for Metabolic Research, VA San Diego Healthcare System and University of California, San Diego, California.

${ }^{2}$ Capital Clinical Research Center, Olympia, Washington.

${ }^{3}$ Pharmapace, Inc., San Diego, California.

${ }^{4}$ AstraZeneca, Gaithersburg, Maryland.
}

(C) Robert R. Henry, et al., 2016; Published by Mary Ann Liebert, Inc. This Open Access article is distributed under the terms of the Creative Commons Attribution Noncommercial License (http://creativecommons.org/licenses/by-nc/4.0/) which permits any noncommercial use, distribution, and reproduction in any medium, provided the original author(s) and the source are credited. 
glucose-lowering therapies (metformin, sulfonylurea, thiazolidinedione, or any two of these agents), comprised a 30week controlled period followed by an open-ended, openlabel extension. All patients in the extension study $(N=258$; $87.5 \%$ of the intent-to-treat [ITT] population) were treated with exenatide QW, either continuing from the controlled period or switching from exenatide twice daily (BID) to exenatide QW. Results from the 6-month and 1-, 2-, 3-, and 5 -year assessments of HbA1c, fasting plasma glucose (FPG), body weight, and adverse events have previously been reported. $^{3-7}$ The purpose of this analysis was to examine the safety, efficacy, and changes in glucose-lowering therapy, and to assess key subpopulations, among patients with T2D treated with exenatide QW, continuously for up to 6 years in the DURATION-1 trial.

\section{Materials and Methods}

\section{Study design}

The study design of DURATION-1 has been reported previously. ${ }^{3}$ Briefly, the original study enrolled patients aged $\geq 16$ years with T2D (HbA1c of 7.1\%-11.0\%) treated for $\geq 2$ months before screening with diet modification and exercise, or pharmacotherapy with metformin, sulfonylurea, thiazolidinedione, or a combination of any two of these agents. Exenatide BID and QW was administered by subcutaneous injection. Patients received exenatide BID $5 \mu \mathrm{g}$ for 3 days before randomization, then were randomized to receive exenatide QW $2 \mathrm{mg}$, or exenatide BID ( $5 \mu \mathrm{g}$ for the first 4 weeks and then $10 \mu \mathrm{g}$ ) through week 30 . At 30 weeks, patients entered an uncontrolled, openlabel, open-ended study phase in which all patients received exenatide QW $2 \mathrm{mg}$. The study was initially conducted with a precommercial grade of exenatide $\mathrm{QW}$, but patients transitioned to commercial-grade exenatide QW during the extension period. Patients were not followed up after discontinuation from the study, but measurements from the final visit were available.

Restrictions on concomitant medication use were eased over time. Medication changes were allowed if deemed necessary by the investigator or primary care physician but had to be reported to the study sponsor. Added glucoselowering medications were identified by Anatomical Therapeutic Chemical codes, and the addition of drugs (not neutral change between brand and generic forms) was verified by an AstraZeneca physician. Data for changes in dose or substitution of medications within a class were not available.

A common clinical protocol was approved for each study site by the appropriate institutional review board. Patients provided written informed consent before study participation. The study was conducted in accordance with the principles described in the Declaration of Helsinki, including all amendments through to the South Africa revision of $1996 .^{8}$

\section{Endpoints}

During the open-ended extension period, patients visited the clinic weekly during weeks 31-34, 2 weeks later at week 36, at 4-week intervals during weeks 40-76, and subsequently at 8week intervals until study end or the early termination visit. Endpoints assessed for each visit during the open-ended extension period included vital signs, height, and weight; HbA1c, FPG (in $\mathrm{mg} / \mathrm{dL}$; divide by 18 for $\mathrm{mmol} / \mathrm{L}$ ), and homeostatic model assessment (HOMA) of $\beta$-cell function (HOMA-B) and insulin sensitivity (HOMA-S); adverse events (including injection-site examination) that were coded according to the Medical Dictionary for Regulatory Activities (version 17.0); and medication review. During the open-ended extension period, assessments at each visit from week 36 onward included anthropometric measures, fasting insulin, proinsulin, glucagon, lipids, anti-exenatide antibodies, chemistry, hematology, and urinalysis. Data are presented for the visits at specific weeks that correspond approximately with years $1-6$.

\section{Statistical analysis}

Efficacy analyses utilized the 6-year completer population, which comprised patients who completed 6 years ( 312 weeks) of treatment, whether they remained active in the study or withdrew after 6 years. The last observation carried forward approach was applied to estimate missing postbaseline values by treatment. For patients who were assigned to receive exenatide BID during the controlled period, available data after week 30 were used to impute subsequent missing data for exenatide QW. Safety analyses utilized the ITT population, comprising individuals who received $\geq 1$ dose of exenatide.

The primary analysis method for changes in HbAlc was analysis of variance (ANOVA; general linear model, including the original treatment assignment, baseline HbA1c stratum, and concomitant sulfonylurea use at screening), as prespecified in the statistical analysis plans for the 30- and 52-week data analyses. To account for individual patient's baseline HbA1c, a secondary analysis was performed using analysis of covariance (ANCOVA; general linear model, including the original treatment assignment, concomitant sulfonylurea use at screening, and baseline HbA1c values). Secondary parameters were analyzed using ANCOVA (general linear model including the original treatment assignment, baseline HbA1c stratum, concomitant sulfonylurea use at screening, and baseline values for the respective parameters). Least-squares means (LSM), standard errors, and 95\% confidence intervals (CIs) were calculated for changes from baseline in efficacy parameters. For adverse events, overall incidences within the ITT population were calculated. Exposure-adjusted incidence among ITT patients receiving exenatide $\mathrm{QW}$ during the controlled and extension periods was also calculated.

Post-hoc analyses were conducted to study the timing of specific events over the study duration. Such analyses assessed the addition of non-GLP-1RA glucose-lowering medications and the timing of key adverse events, including nausea, injection-site reactions, hypoglycemia with and without sulfonylureas and insulin, and serious adverse events.

Post-hoc analyses also examined patient subpopulations of interest. These key patient subpopulations included patients with no changes or additions to concomitant glucose-lowering medications over 6 years; patients who discontinued before completing 6 years of treatment (noncompleters); and patients with systolic blood pressure (BP) $>130 \mathrm{mmHg}$ at baseline (for $\mathrm{BP}$ changes). Where calculation of inferential statistics was appropriate, the ANCOVA model described above was used.

\section{Results}

The ITT population comprised 295 patients (Fig. 1A); of these, 258 patients $(87.5 \%)$ continued in the extension period and $136(46 \%)$ completed at least 6 years of treatment and were included in the 6-year completer population (Fig. 1B). 
Demographics and baseline characteristics in the ITT and 6-year completer and noncompleter populations are shown in Table 1. Compared to noncompleters, completers were slightly older $(P=0.016)$, had a lower baseline HbA1c $(P=0.014)$, and were more often white $(P=0.034)$ (Table 1$)$. All other baseline characteristics were comparable between completers and noncompleters.

Of the 136 completers, 78 (57\%) added no new glucoselowering medications during the 6-year follow-up period (Fig. 1C). At screening and at endpoint, metformin and sulfonylureas were the most commonly used concomitant glucose-lowering therapies. Use of metformin and sulfonylureas appeared to increase during the trial, whereas use of thiazolidinediones appeared to decrease (Fig. 1D). Addition of insulin was observed after 12 months of treatment. Insulin use was less frequent than use of oral therapies (fast-acting, up to $2.9 \%$; intermediate-acting, $0.7 \%$; long-acting, $8.8 \%$ ). Few patients received dipeptidyl peptidase- 4 inhibitors (up to $2.9 \%$ ) or bromocriptine $(0.7 \%)$ and none used sodiumglucose cotransporter-2 inhibitors.

\section{Glycemic measures}

Significant improvements in HbAlc from baseline were observed in the 6-year completer population at each annual time point, including at 6 years, as assessed by ANOVA (LSM change, $-1.6 \%$ [95\% CI, -1.8, -1.4]; Fig. 2A), the primary outcome measure. Significant HbA1c improvements from baseline were also seen by ANCOVA (LSM change, $-1.1 \%$ [95\% CI, -1.2, -0.9]; Supplementary Fig. S1; Supplementary Data are available at www.liebertonline.com/dia).
At years $1,2,3,4,5$, and $6, \mathrm{HbA} 1 \mathrm{c}<7.0 \%$ was achieved by $79.4 \%, 63.2 \%, 60.3 \%, 59.6 \%, 46.3 \%$, and $46.3 \%$ of 6 -year completers, respectively, and $\mathrm{HbA} 1 \mathrm{c} \leq 6.5 \%$ was achieved by $63.2 \%, 43.4 \%, 35.3 \%, 39.7 \%, 31.6 \%$, and $33.1 \%$, respectively. At each annual time point, including 6 years, 6-year completers had significant improvements from baseline in FPG (LSM change, $-28 \mathrm{mg} / \mathrm{dL}$ [95\% CI, -37, -18]; Fig. 2B). The 6-year completer population also demonstrated improvements from baseline over time in $\beta$-cell function and insulin sensitivity as measured by HOMA (HOMA-B and HOMA-S, respectively). The HOMA-B score was increased from baseline by $78 \%$ at year 1 and subsequently declined but remained elevated relative to baseline (21\% at year 6; Supplementary Table S1). The HOMA-S score was increased from baseline at each annual time point (by $28 \%$ at year 6; Supplementary Table S1).

\section{Cardiovascular risk factors}

Body weight significantly improved from baseline at each annual time point and after 6 years (LSM change, $-4.2 \mathrm{~kg}$ [95\% CI, -5.8, -2.6]; Fig. 2C). At 6 years, significant improvements in multiple measures of dyslipidemia from baseline were also observed (Supplementary Table S1), including total cholesterol (LSM change, $-10.1 \mathrm{mg} / \mathrm{dL}$ [95\% CI, -17.0 , -3.1]), low-density lipoprotein (LDL) cholesterol (LSM change, $-9.8 \mathrm{mg} / \mathrm{dL}$ [95\% CI, -15.6, -4.0]), high-density lipoprotein (HDL) cholesterol (LSM change, $+2.6 \mathrm{mg} / \mathrm{dL}[95 \%$ CI, 0.7, 4.5]), LDL/HDL cholesterol ratio (median change, -0.2 [range, $-2.1,+3.4]$ ), and triglycerides (median change, $-8.0 \mathrm{mg} / \mathrm{dL}$ [range, $-307,+431]$ ). Reductions from baseline in

Table 1. Demographics and Baseline Characteristics

\begin{tabular}{|c|c|c|c|}
\hline & ITT population $(\mathrm{N}=295)$ & 6-Year completers $(\mathrm{n}=136)$ & 6-Year noncompleters $(\mathrm{n}=159)$ \\
\hline Male & $157(53.2)$ & $73(53.7)$ & $84(52.8)$ \\
\hline Age at consent, ${ }^{\text {a }}$ years & $55.0 \pm 9.7$ & $56.5 \pm 8.5$ & $53.8 \pm 10.5$ \\
\hline \multicolumn{4}{|l|}{ Race/ethnicity ${ }^{\mathrm{b}}$} \\
\hline White & $230(78.0)$ & $114(83.8)$ & $116(73.0)$ \\
\hline Black & $28(9.5)$ & $15(11.0)$ & $13(8.2)$ \\
\hline Hispanic & $36(12.2)$ & $6(4.4)$ & $30(18.9)$ \\
\hline Asian & $1(0.3)$ & $1(0.7)$ & $0(0.0)$ \\
\hline Duration of $\mathrm{T} 2 \mathrm{D}$, years & $6.7 \pm 5.0$ & $7.1 \pm 5.7$ & $6.4 \pm 4.3$ \\
\hline HbA1c, ${ }^{\text {\% } \%}$ & $8.30 \pm 0.99$ & $8.14 \pm 0.93$ & $8.43 \pm 1.03$ \\
\hline $\mathrm{FPG}, \mathrm{mg} / \mathrm{dL}$ & $169.4 \pm 42.9$ & $165.7 \pm 41.0$ & $172.6 \pm 44.3$ \\
\hline Body weight, kg & $101.8 \pm 19.9$ & $101.1 \pm 17.5$ & $102.5 \pm 21.8$ \\
\hline \multicolumn{4}{|c|}{ T2D management method at screening } \\
\hline SU-based & $109(36.9)$ & $53(39.0)$ & $56(35.2)$ \\
\hline SU only & $16(5.4)$ & $9(6.6)$ & $7(4.4)$ \\
\hline SU+metformin & $82(27.8)$ & $38(27.9)$ & $44(27.7)$ \\
\hline SU+TZD & $10(3.4)$ & $5(3.7)$ & $5(3.1)$ \\
\hline SU+metformin+TZD & $1(0.3)$ & $1(0.7)$ & $0(0.0)$ \\
\hline Non-SU based & $186(63.1)$ & $83(61.0)$ & $103(64.8)$ \\
\hline Diet and exercise & $43(14.6)$ & $17(12.5)$ & $26(16.4)$ \\
\hline Metformin only & $106(35.9)$ & $48(35.3)$ & $58(36.5)$ \\
\hline TZD only & $9(3.1)$ & $7(5.1)$ & $2(1.3)$ \\
\hline Metformin+TZD & $28(9.5)$ & $11(8.1)$ & $17(10.7)$ \\
\hline
\end{tabular}

Data are $n(\%)$ or mean \pm standard deviation.

${ }^{\mathrm{a}} P<0.05$ for completers versus noncompleters.

${ }^{\mathrm{b}}$ Percentages may not total $100 \%$ due to rounding.

${ }^{\mathrm{c}} P<0.05$ for the proportion of white patients for completers versus noncompleters.

FPG, fasting plasma glucose; HbAlc, glycated hemoglobin; ITT, intent-to-treat; SU, sulfonylurea; T2D, type 2 diabetes; TZD, thiazolidinedione. 


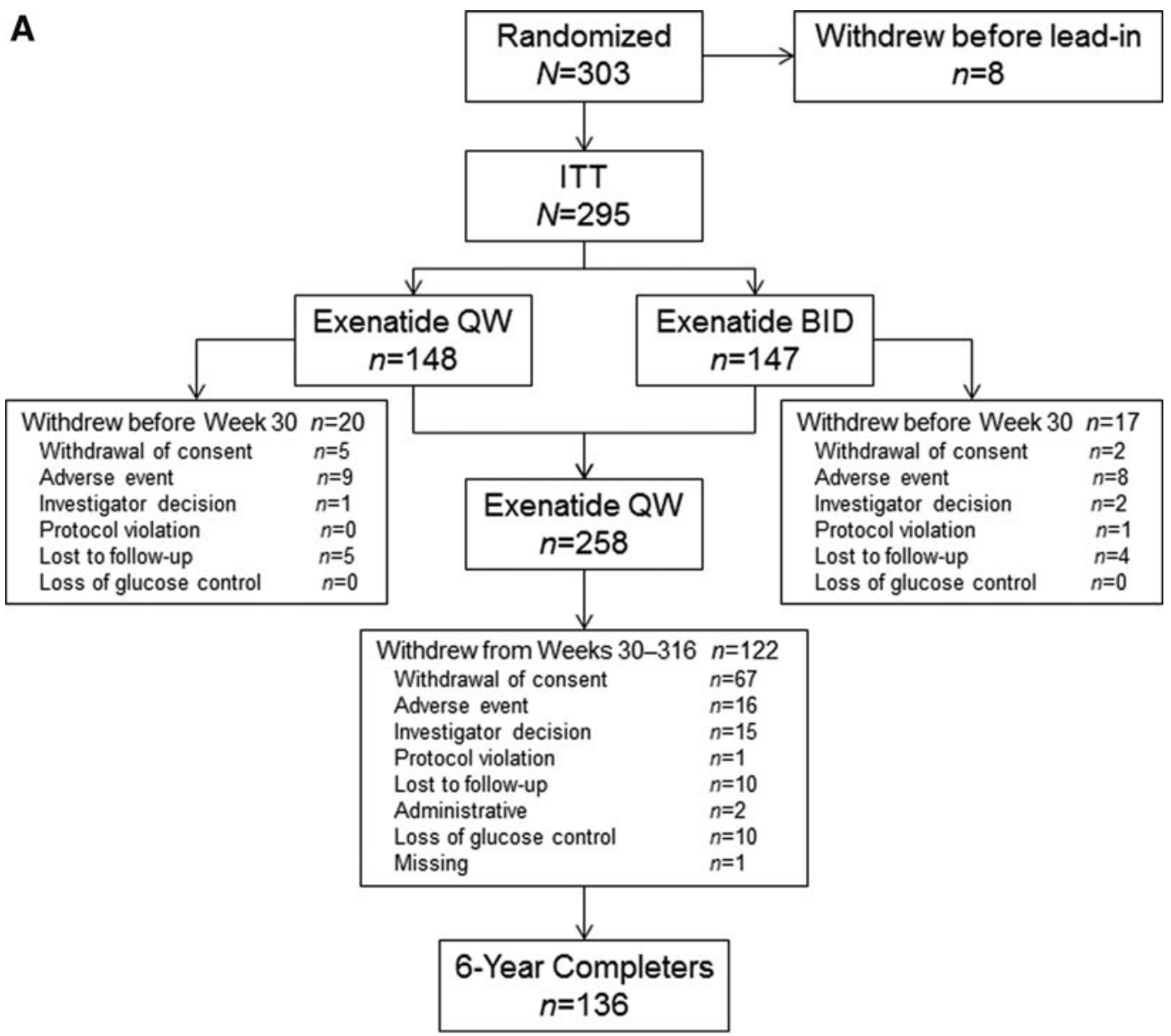

B

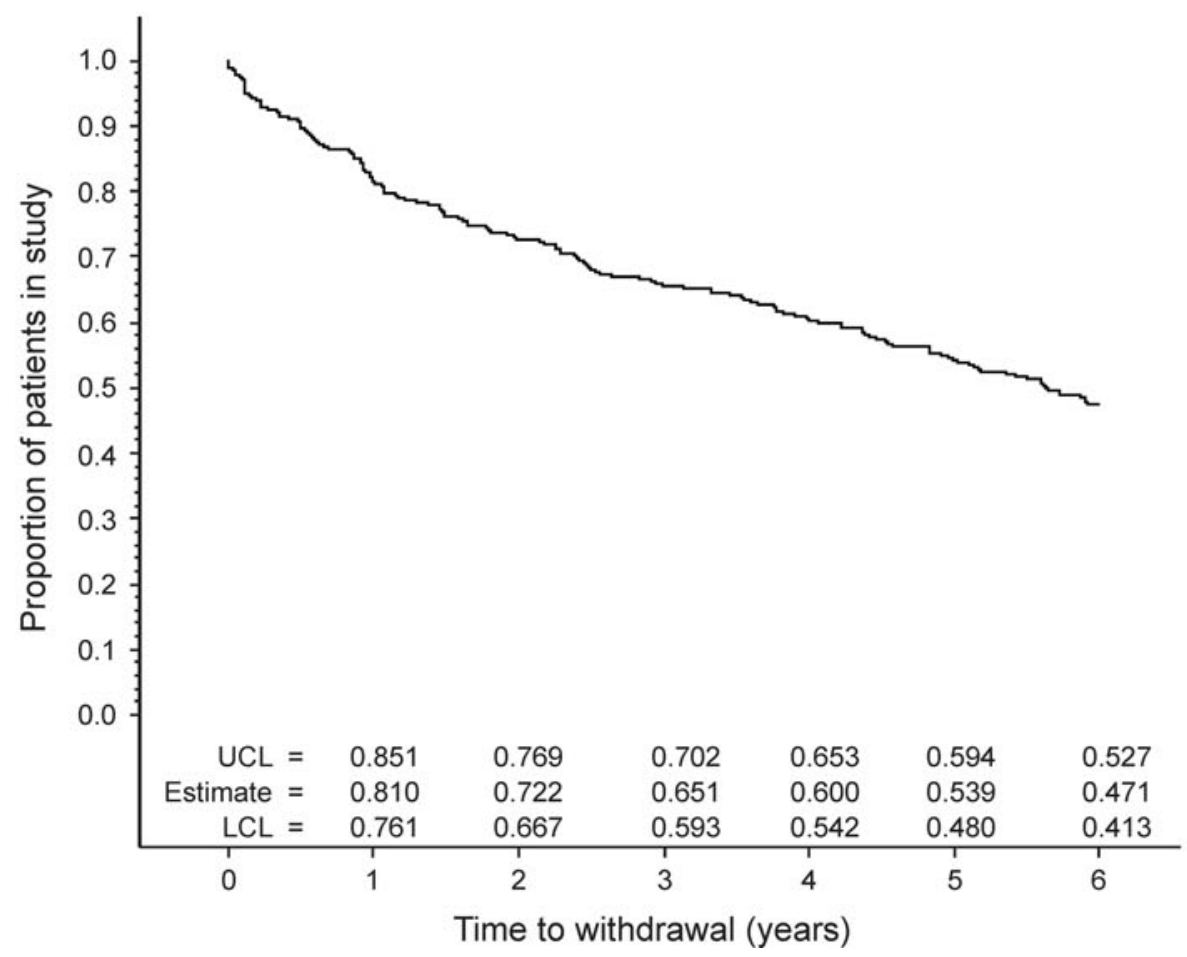

FIG. 1. (continued next page) 

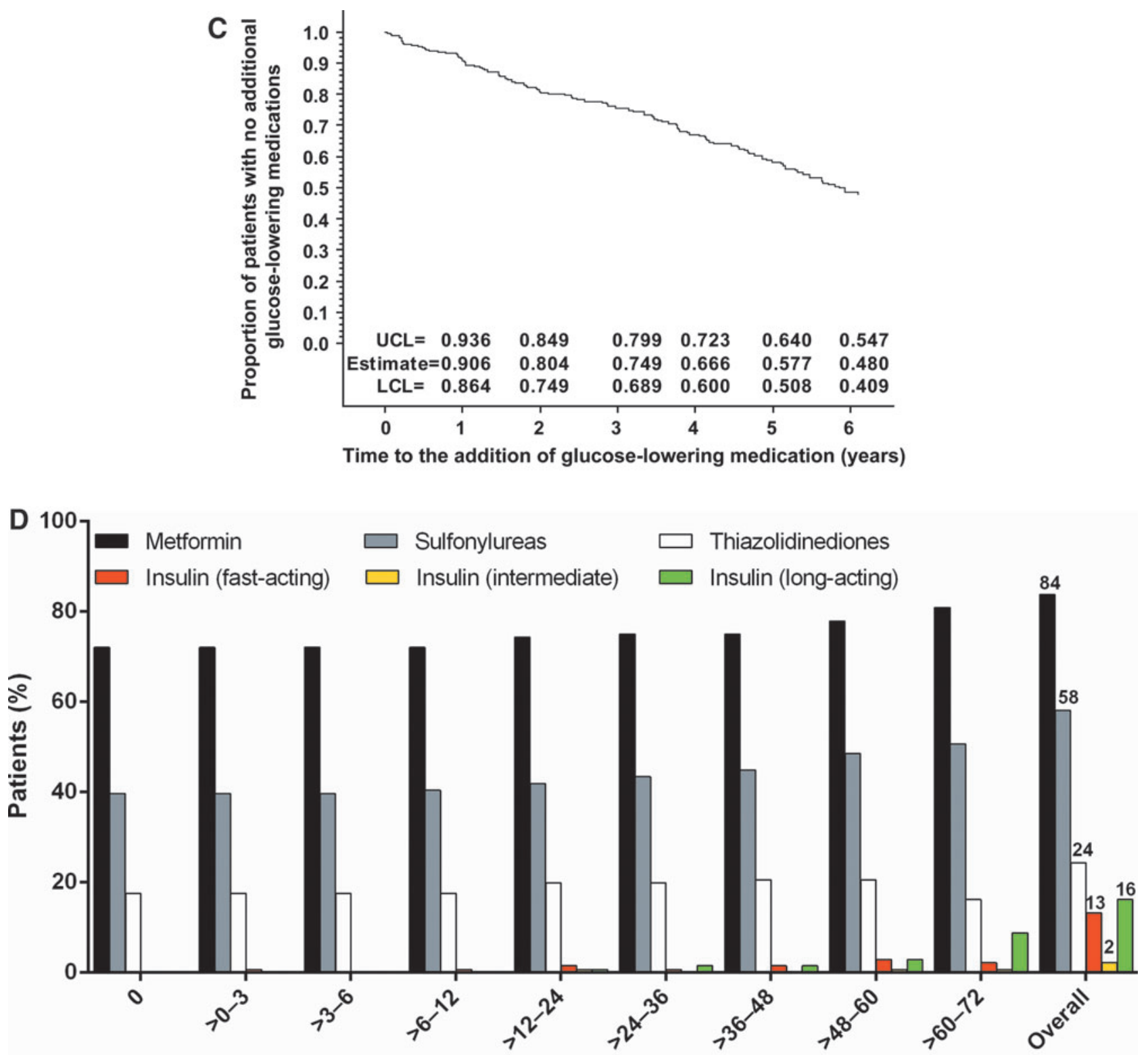

Time period (months)

FIG. 1. (A) Patient disposition. (B) Kaplan-Meier plot of time to withdrawal and estimates of proportion of patients remaining in study. (C) Kaplan-Meier plot of time to addition of new glucose-lowering medication. (D) Time course of use of the most common concomitant glucose-lowering therapies in 6-year completers $(n=136)$. Point estimates and 95\% confidence intervals for Kaplan-Meier plots were calculated for the time the first event was observed in each year. BID, twice daily; ITT, intent-to-treat; LCL, lower confidence limit; QW, once weekly; UCL, upper confidence limit.

systolic and diastolic BP were observed in the completer population but were not maintained throughout the study (Supplementary Table S1). The subgroup of patients with baseline systolic $\mathrm{BP} \geq 130 \mathrm{mmHg}$ had reductions from baseline in systolic and diastolic BP that were significant at most annual time points through to 6 years (LSM [95\% CI], $-9.9[-15.4$, $-4.4] /-5.8[-9.1,-2.4] \mathrm{mmHg}$ ).

\section{Patients with no additional glucose-lowering medications}

Six-year completers who received no additional glucoselowering medications had significant reductions from base- line in $\mathrm{HbA} 1 \mathrm{c}$ at each annual time point, including at 6 years, as assessed by ANOVA (LSM change, $-1.8 \%$ [95\% CI, -2.1, -1.6]; Fig. 2A) and ANCOVA (LSM change, $-1.3 \%$ [95\% CI, $-1.5,-1.1]$; Supplementary Fig. S1). At 6 years, HbA1c goals of $<7.0 \%$ and $\leq 6.5 \%$ were achieved by $55.1 \%$ and $41.0 \%$ of patients, respectively. Similarly, this subgroup had significant reductions from baseline in FPG at each annual time point through to 6 years (LSM change, $-26.0 \mathrm{mg} / \mathrm{dL}$ [95\% CI, $-35.0,-16.9]$; Fig. 2B). Reductions in body weight observed in this subgroup were numerically greater than in the overall cohort (LSM change at 6 years, $-6.1 \mathrm{~kg}$ [95\% CI, $-7.5,-4.6])$ and more stable over time (Fig. 2C). 


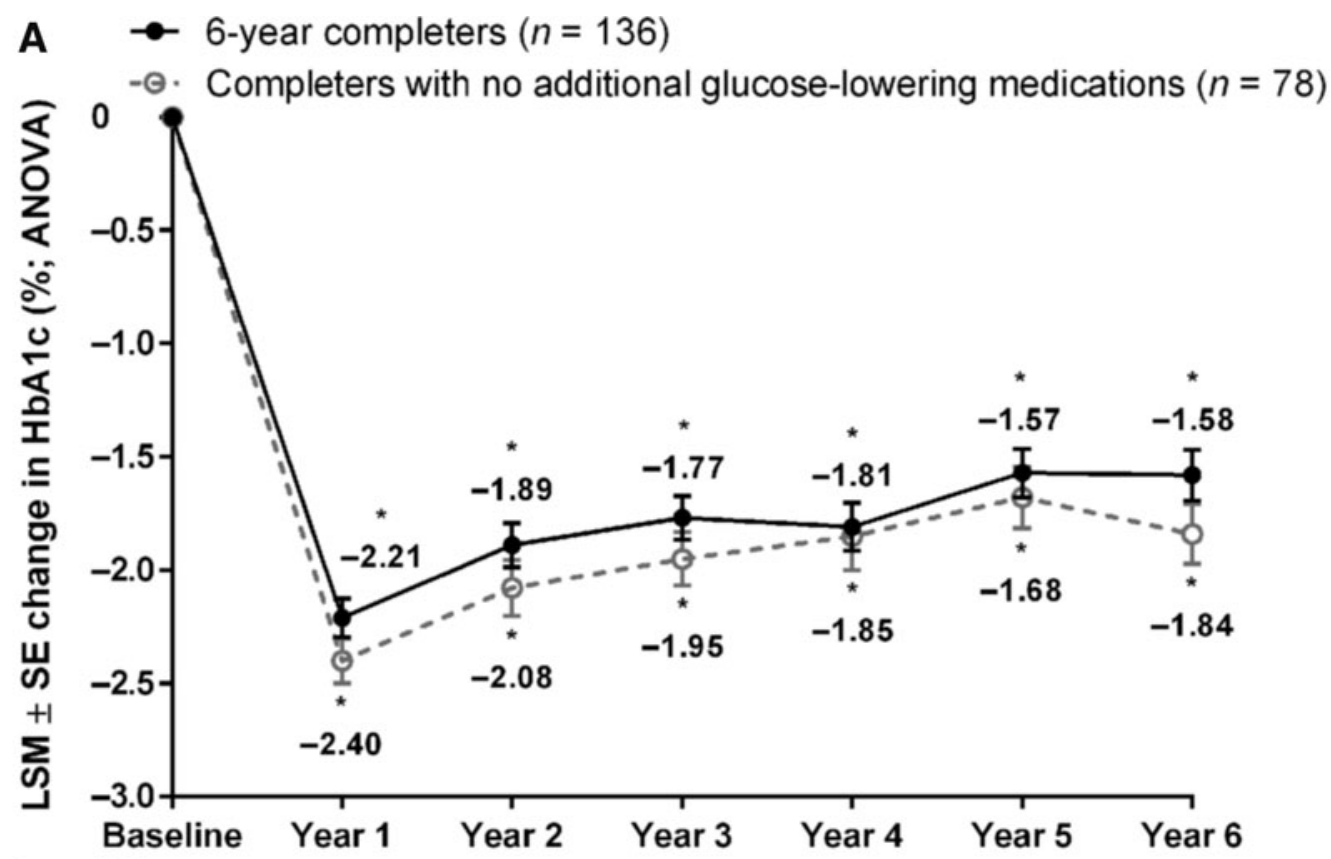

Absolute values (\%)

$\begin{array}{llllllll}\text { Completers } & 8.14 & 6.49 & 6.81 & 6.90 & 6.81 & 7.01 & 7.07 \\ \text { Completers, no } & 8.16 & 6.38 & 6.63 & 6.71 & 6.72 & 6.84 & 6.86\end{array}$
medications added

B $\rightarrow$ 6-year completers $(n=136)$

- $\Theta$. Completers with no additional glucose-lowering medications $(n=78)$

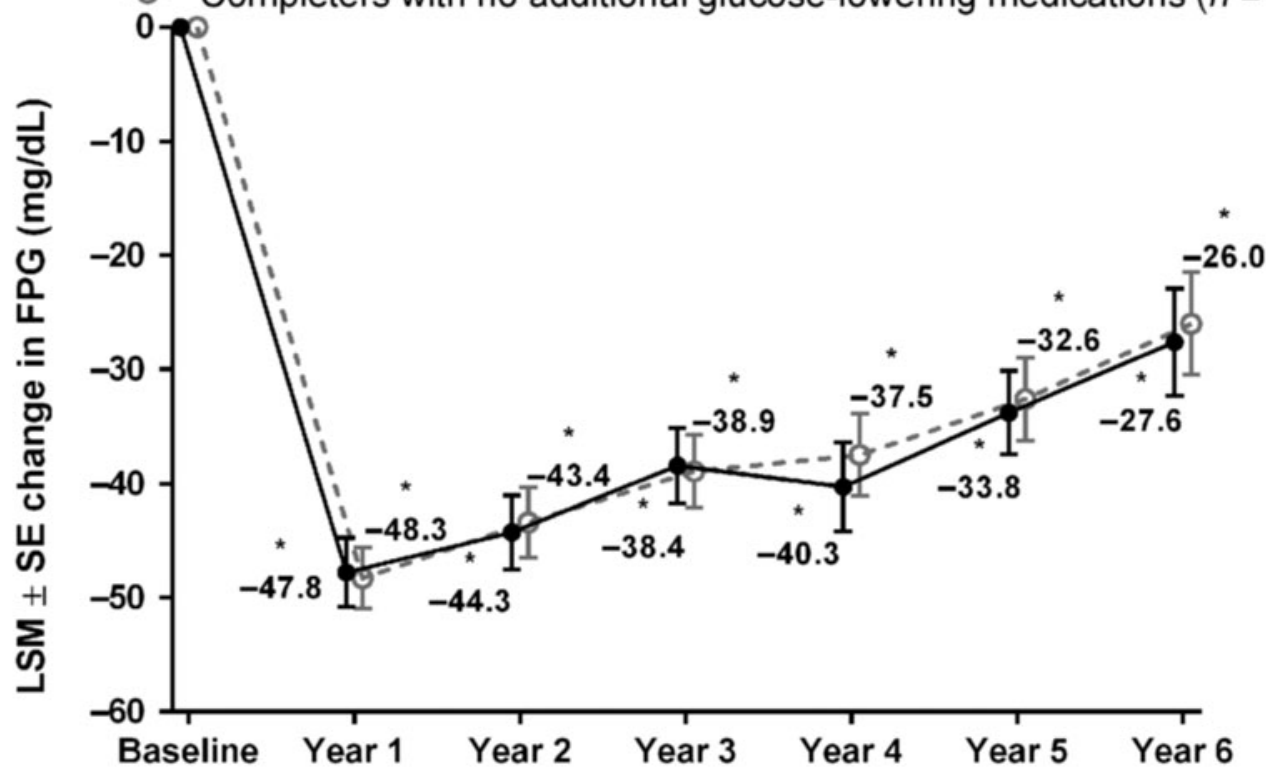

Absolute values $(\mathrm{mg} / \mathrm{dL})$

$\begin{array}{lrrrrrrr}\text { Completers } & 165.7 & 121.6 & 125.3 & 127.3 & 128.3 & 133.3 & 142.0 \\ \text { Completers, no } & 164.2 & 116.0 & 120.9 & 124.6 & 126.6 & 131.7 & 137.6\end{array}$

medications added

FIG. 2. (continued next page) 


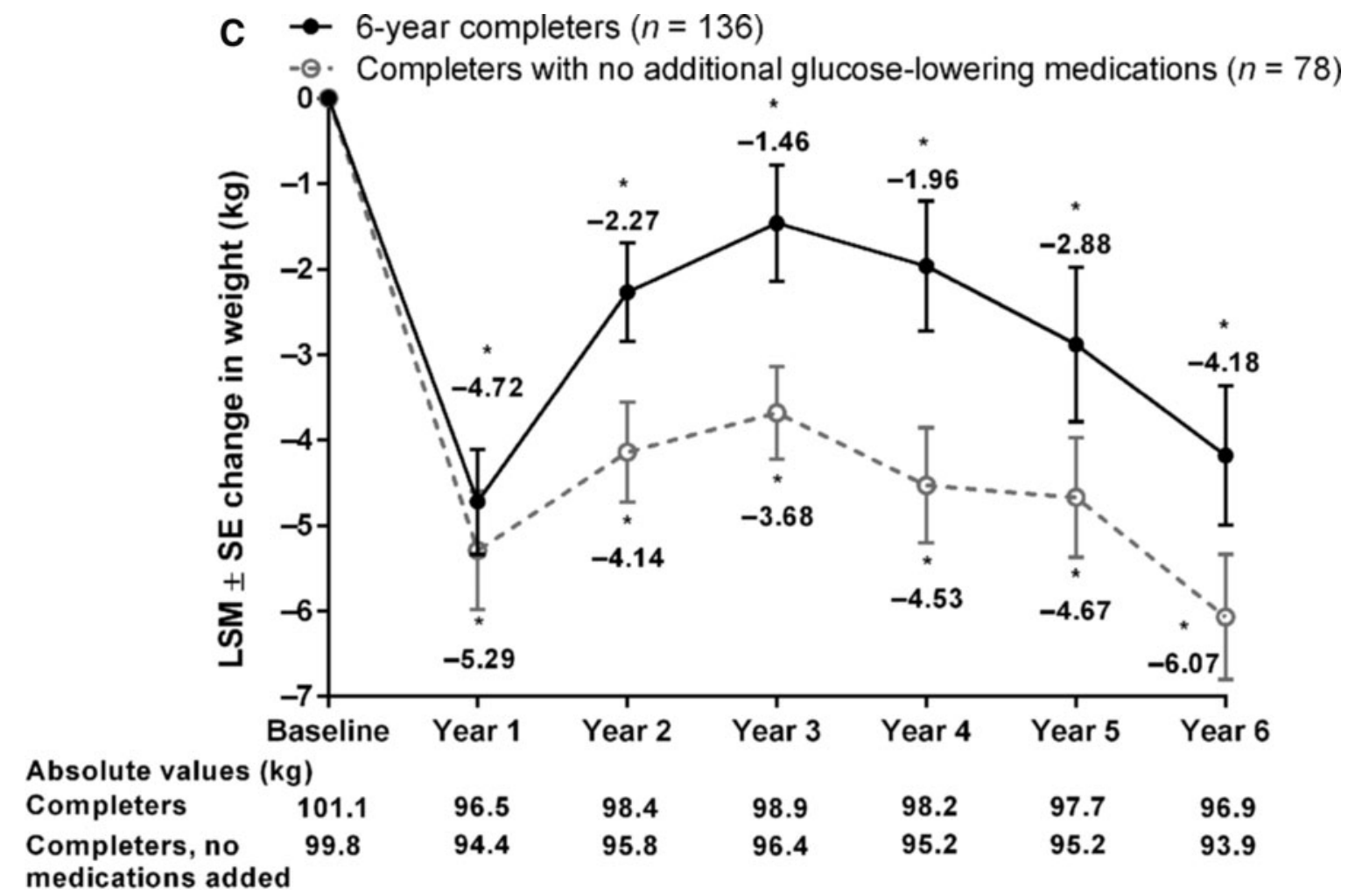

FIG. 2. Key efficacy parameters in all 6-year completers $(n=136)$ and those not on additional glucose-lowering medications $(n=78)$. LSM \pm SE changes from baseline in (A) HbA1c by ANOVA, (B) FPG, and (C) body weight. $* P<0.05$ for change from baseline. ANOVA, analysis of variance; FPG, fasting plasma glucose; HbA1c, glycated hemoglobin; LSM, least-squares mean; SE, standard error.

Improvements in levels of total cholesterol (LSM change, $-13.2 \mathrm{mg} / \mathrm{dL}[95 \% \mathrm{CI},-20.9,-5.5]), \mathrm{LDL}$ cholesterol (LSM change, $-10.9 \mathrm{mg} / \mathrm{dL}[95 \% \mathrm{CI},-17.7,-4.0])$, HDL cholesterol (LSM change, $2.7 \mathrm{mg} / \mathrm{dL}$ [95\% CI, 0.6, 4.7]), and triglycerides (median change, $-17.0 \mathrm{mg} / \mathrm{dL}$ [range, $-307,+149]$ ) were also observed at 6 years among 6-year completers who received no additional glucose-lowering medications; however, reductions in systolic and diastolic BP were nonsignificant, with LSM changes of $-0.2 \mathrm{mmHg}(95 \% \mathrm{CI},-3.4,2.9)$ and $-1.4 \mathrm{mmHg}$ (95\% CI, $-3.3,0.5)$, respectively.

Of the 78 completers who received no additional glucoselowering medications, $24(30.8 \%)$ reported nausea during treatment with exenatide QW during the 6 years of follow-up.

\section{Patients who discontinued therapy}

The mean time to discontinuation of study treatment in 6year noncompleters was 903 days (2.5 years). Six-year noncompleters were observed to respond to therapy, with a mean ( \pm standard deviation) change in $\mathrm{HbA} 1 \mathrm{c}$ of $-0.7 \% \pm 1.4 \%$, FPG of $-14 \pm 58 \mathrm{mg} / \mathrm{dL}$, and body weight of $-2.7 \pm 6.3 \mathrm{~kg}$ at study endpoint (i.e., each patient's last measurement in the study). Of the 159 noncompleters, 64 (40.3\%) reported nausea adverse events during the initial 30 -week assessment period and $36(22.6 \%)$ reported nausea during the openended assessment period.

\section{Safety and tolerability}

In this subgroup of 136 patients choosing to continue on therapy, no incremental or unexpected safety findings were observed with long-term exenatide QW treatment. Treatment-emergent adverse events with an incidence of $\geq 10 \%$ over the open-ended extension period are shown in Table 2. Nausea (mostly mild) was the most common adverse event with exenatide QW during the initial controlled period. Subsequently, the incidence of nausea decreased (from 0.846 to 0.076 events/year), as did the incidence of injection-site pruritus (from 0.510 to 0.016 events/year) and injection-site erythema (from 0.137 to 0.013 events/year).

Serious adverse events were reported in 58 ITT patients $(22.5 \%)$ during the extension period (ranging from $4.0 \%$ to $8.7 \%$ in the 6-month intervals during the open-ended assessment period; Supplementary Table S2). Treatmentemergent adverse events leading to withdrawal were infrequent $(6.2 \%)$ during the extension period. The adverse events leading to withdrawal in $>1$ patient were nausea $(n=2)$ and T2D progression (loss of glucose control; $n=2$ ).

Adverse events of special interest were rare during the 6 years of follow-up: one case of pancreatitis (annual rate of 0.001 events/year; considered related to treatment and led to withdrawal), one case of pancreatic carcinoma (not considered related to treatment and led to withdrawal), and two cases of acute renal failure (not considered related to treatment and did not lead to withdrawal) were reported.

No major hypoglycemia events were reported. Most minor hypoglycemia events over 6 years occurred with concomitant sulfonylurea use; $28.6 \%$ of patients with sulfonylurea use at screening and $6.4 \%$ with no sulfonylurea use reported $\geq 1$ minor hypoglycemia event. The time course of nausea 
Table 2. Treatment-Emergent Adverse Events with an Incidence of $\geq 10 \%$ Reported in Patients Receiving Exenatide Once Weekly During the 30-Week or Open-Ended Assessments Through 6 Years (INTENT-To-Treat Population)

\begin{tabular}{|c|c|c|c|c|}
\hline \multirow[b]{2}{*}{ Adverse event } & \multicolumn{2}{|c|}{ 30-Week assessment $(\mathrm{n}=148)$} & \multicolumn{2}{|c|}{ Open-ended assessment $(\mathrm{n}=278)$} \\
\hline & Incidence, \% & $\begin{array}{l}\text { Annual event } \\
\text { rate, events/year }\end{array}$ & Incidence, \% & $\begin{array}{l}\text { Annual event } \\
\text { rate, events/year }\end{array}$ \\
\hline Upper respiratory tract infection & 8.1 & 0.162 & 41.9 & 0.172 \\
\hline Nasopharyngitis & 6.8 & 0.187 & 28.3 & 0.160 \\
\hline Diarrhea & 16.2 & 0.373 & 26.0 & 0.102 \\
\hline Sinusitis & 4.7 & 0.087 & 21.7 & 0.104 \\
\hline Arthralgia & 4.7 & 0.124 & 19.0 & 0.067 \\
\hline Back pain & 4.7 & 0.087 & 18.6 & 0.057 \\
\hline Urinary tract infection & 10.1 & 0.224 & 18.2 & 0.076 \\
\hline Nausea & 27.0 & 0.846 & 17.1 & 0.076 \\
\hline Pain in extremity & 0.7 & 0.025 & 15.9 & 0.050 \\
\hline Vomiting & 10.8 & 0.361 & 15.5 & 0.069 \\
\hline Hypertension & 3.4 & 0.062 & 15.1 & 0.043 \\
\hline Bronchitis & 2.7 & 0.050 & 14.3 & 0.047 \\
\hline Musculoskeletal pain & 1.4 & 0.025 & 13.6 & 0.042 \\
\hline Cough & 3.4 & 0.062 & 10.5 & 0.032 \\
\hline Gastroenteritis, viral & 8.1 & 0.149 & 10.1 & 0.033 \\
\hline Constipation & 10.1 & 0.199 & 9.7 & 0.028 \\
\hline Injection-site pruritus & 18.2 & 0.510 & 5.4 & 0.016 \\
\hline
\end{tabular}

showed a decreased incidence after the initial controlled period, while the incidence of hypoglycemia initially decreased but subsequently increased (Supplementary Fig. S2).

Small mean ( \pm standard deviation) increases from baseline in the heart rate were seen throughout the 6-year observation period (2.1 [9.6] beats/min at 6 years), but the magnitude of the change did not increase over time (Supplementary Table S1).

\section{Discussion}

Long-term data on the efficacy and tolerability of treatment for T2D provide important information for both physicians and patients. Results of this analysis show that, over 6 years of treatment, patients treated with exenatide QW who remained on therapy demonstrated sustained improvements from baseline in clinical parameters relating to glycemic control, measures of $\beta$-cell function and insulin sensitivity, and cardiovascular risk factors. In addition, a considerable proportion of these patients consistently achieved HbA1c goals, satisfying therapeutic guidelines. These data also showed no new pattern or increased incidence of adverse events over time on therapy.

To date, few glucose-lowering therapies have demonstrated efficacy and safety out to 6 years, although 2- and 3year analyses have been conducted for several GLP-1RAs, including exenatide BID and QW. In the Liraglutide Effect and Action in Diabetes (LEAD)-3 trial, in which $43 \%$ of patients completed 2 years of treatment, the mean changes from baseline in $\mathrm{HbA} 1 \mathrm{c}$ were $-0.9 \%$ and $-1.1 \%$ for liraglutide 1.2 and $1.8 \mathrm{mg}$, respectively. ${ }^{9}$ In the HARMONY 3 study, $68 \%$ of patients completed 2 years of treatment with albiglutide (including $26 \%$ of completers who received hyperglycemic rescue therapy), leading to a mean change from baseline in $\mathrm{HbA} 1 \mathrm{c}$ of $-0.63 \% .^{10}$ In a long-term extension study of exenatide BID, the mean change from baseline in
HbA1c was $-1.0 \%$ in patients completing 3 years of treatment $(41 \%) .{ }^{11}$ In the $63 \%$ of patients who completed 3 years of treatment with exenatide QW in the DURATION-3 study, the mean change from baseline in HbAlc was $-1.1 \%{ }^{12}$ The mean change in $\mathrm{HbA} 1 \mathrm{c}$ was also $-1.1 \%$ in a pooled analysis of patients treated with exenatide QW for 2.5-3 years in the DURATION-1, -2 , and -3 trials. ${ }^{13}$ Thus, these findings contribute to the small number of studies demonstrating longterm efficacy with GLP-1RA treatment.

The investigator and patient investment in such a longterm study should not be underestimated, with over 50 study visits and measurements of $\mathrm{HbAlc}$ and other parameters per patient during the 6 years of follow-up. Accordingly, only $46 \%$ of the initial ITT population completed 6 years of treatment. This context must be considered for proper interpretation of the results. For example, although $\mathrm{HbA} 1 \mathrm{c}<7.0 \%$ was achieved by $63(46.3 \%) 6$-year completers, this amounts to $21.4 \%$ of the overall ITT population. Furthermore, it is not surprising that some patients would have had additions to their glucose-lowering therapy regimens over this long study duration, although over half of the 6-year completers did not receive any additional glucose-lowering therapies during the study, and changes in glycemic parameters in patients without such additions were similar to those in completers overall.

Given the progressive nature of T2D, it would be expected to see indications of disease progression during a study of this duration. Initial mean improvements in HOMA-B were attenuated over the course of the study, although improvement was still observed with exenatide QW at 6 years. Similarly, increases were observed following the initial mean reduction in FPG, suggesting gradual loss of glycemic control. Although the patients remaining on treatment for 6 years still had progressive T2D, most managed their hyperglycemia without insulin.

Since the completer population is a self-selected population of patients who continued therapy, it may be suggested 
that its results are influenced by withdrawal of patients who experienced inadequate glucose control or adverse events. Analysis of the last data available for patients who discontinued shows that mean reductions in HbA1c and FPG were lower in noncompleters than in 6-year completers, and 10 patients had documented withdrawal due to loss of glucose control over the 6 years. Similarly, the incidence of nausea in noncompleters during the initial 30-week assessment (40\%) was higher than that in completers (24\%). Overall, 33 patients withdrew due to adverse events during the course of the study, most often due to nausea. However, given the nature and duration of this extension trial, it is equally possible that patients discontinued for personal reasons such as lack of time or interest, relocation, or trial fatigue, consistent with the "withdrawal of consent" designation that was the most common reason for discontinuation.

Significant mean body weight reductions of $\geq 1.5 \mathrm{~kg}$ were observed from years 1-6, although the degree of weight loss was diminished in the middle years of assessment. This may be attributable, at least in part, to changes to concomitant glucose-lowering medication regimens in some patients, although changes in patient compliance with dietary advice were not recorded. The mean weight loss observed in the 6year completers who had no additions to their glucoselowering regimen during the course of follow-up was greater and more stable than in the overall cohort of completers.

Previous short-term studies suggest that common adverse events primarily occur early in therapy with GLP1RAs. ${ }^{14,15}$ No incremental safety findings were observed with up to 6 years of exenatide QW treatment in the current study of a self-selected population. The exposure-adjusted event rates of nausea and injection-site reactions with exenatide QW were lower during the extension period than during the 30 -week controlled period. The incidence of hypoglycemia remained consistently low over the course of the study. A small increase in heart rate (approximately 2-3 beats/min) was also noted. Although this has been observed for multiple GLP-1RAs, the mechanism for this effect is unclear. ${ }^{16}$

The nature of this follow-up analysis is associated with limitations. There was no control group in the extension phase, and the open-label study design has the potential to affect expectations and perceptions of treatment and to create bias. Also, the efficacy analysis was conducted on the completer population, a nonrandomized subset of the ITT population. Although this approach may represent a subset of patients who responded well to treatment during the initial controlled study period or were more adherent, it was deemed to be the most informative analysis for the nature and duration of this long-term study. Although the subgroup of patients with no additions to glucose-lowering therapies had greater and more stable weight loss, we were unable to explore the influence of discontinuing concomitant therapies; in particular, some weight loss may have occurred in patients who discontinued thiazolidinediones or sulfonylureas. Changes in practice may have affected the trial; due to new clinical perspectives regarding cardiovascular profiles of T2D treatments, routine use of thiazolidinediones decreased during the course of the trial, ${ }^{17}$ potentially contributing, at least partially, to the gradual mean weight loss after year 3 . Data were not collected on patient dieting or other lifestyle changes that could have influenced body weight findings.
Further information on the long-term efficacy of exenatide QW will be explored in the ongoing Exenatide Study of Cardiovascular Event Lowering (EXSCEL) Trial (ClinicalTrials.gov identifier: NCT01144338), which will compare the effects of usual care with or without exenatide QW on major cardiovascular outcomes in $\sim 14,000$ patients with T2D over an anticipated duration of up to 7.5 years.

\section{Conclusions}

In this long-term extension study, treatment with exenatide QW in $46 \%$ of patients who chose to remain in the trial over 6 years was associated with clinically significant, sustained improvements in glycemic control and weight without unexpected safety findings. These data support the efficacy and safety of long-term therapy with exenatide QW.

\section{Acknowledgments}

Elise Hardy, MD, of AstraZeneca assisted with verifying data on additions of glucose-lowering medications during the study. Sushma Soni of inScience Communications, Springer Healthcare (Philadelphia, PA), provided medical writing support, which was funded by AstraZeneca. Mary Beth DeYoung, $\mathrm{PhD}$, of AstraZeneca critically reviewed the manuscript. This study was initiated by Amylin Pharmaceuticals LLC and subsequently supported by AstraZeneca and BristolMyers Squibb.

\section{Authors' Contributions}

R.R.H. and E.J.K. participated in data collection and interpretation, conceiving post-hoc analyses, and preparing and critically revising the manuscript. J.H. participated in data analysis and preparing and critically revising the manuscript. N.I. participated in data interpretation and preparing and critically revising the manuscript. All authors reviewed and approved the final version of the manuscript.

\section{Author Disclosure Statement}

R.R.H. has received research grants from Abbott, AbbVie, and Eli Lilly Pharmaceuticals, has served as a consultant or scientific advisory board member, and received honoraria from Amgen Pharmaceuticals, Boehringer Ingelheim, Eli Lilly Pharmaceuticals, Intarcia, Isis Pharmaceuticals, Ligand, Merck, Novo Nordisk, and Sanofi-Aventis. E.J.K. has received research support from Antares Pharma, Asahi Kasei, AstraZeneca, Bristol-Myers Squibb, Catabasis Pharmaceuticals, and Novo Nordisk, and has served on the speaker's bureau for AstraZeneca and Janssen Pharmaceuticals. J.H. is an employee of Pharmapace, Inc. and was an employee of Bristol-Myers Squibb at the time of data collection. N.I. is an employee of AstraZeneca.

\section{References}

1. U.K. Prospective Diabetes Study Group: U.K. prospective diabetes study 16 . Overview of 6 years' therapy of type II diabetes: a progressive disease. Diabetes 1995;44:1249_ 1258.

2. Inzucchi SE, Bergenstal RM, Buse JB, et al.: Management of hyperglycemia in type 2 diabetes, 2015: a patientcentered approach: update to a position statement of the 
American Diabetes Association and the European Association for the Study of Diabetes. Diabetes Care 2015;38: 140-149.

3. Buse JB, Drucker DJ, Taylor KL, et al.: DURATION-1: exenatide once weekly produces sustained glycemic control and weight loss over 52 weeks. Diabetes Care 2010;33: 1255-1261.

4. Taylor K, Gurney K, Han J, et al.: Exenatide once weekly treatment maintained improvements in glycemic control and weight loss over 2 years. BMC Endocr Disord 2011; 11:9.

5. MacConell L, Pencek R, Li Y, et al.: Exenatide once weekly: sustained improvement in glycemic control and cardiometabolic measures through 3 years. Diabetes Metab Syndr Obes 2013;6:31-41.

6. Wysham CH, MacConell LA, Maggs DG, et al.: Five-year efficacy and safety data of exenatide once weekly: longterm results from the DURATION-1 randomized clinical trial. Mayo Clin Proc 2015;90:356-365.

7. Drucker DJ, Buse JB, Taylor K, et al.: Exenatide once weekly versus twice daily for the treatment of type 2 diabetes: a randomised, open-label, non-inferiority study. Lancet 2008;372:1240-1250.

8. World Medical Association: World Medical Association Declaration of Helsinki. Recommendations guiding physicians in biomedical research involving human subjects. JAMA 1997;277:925-926.

9. Garber A, Henry RR, Ratner R, et al.: Liraglutide, a oncedaily human glucagon-like peptide 1 analogue, provides sustained improvements in glycaemic control and weight for 2 years as monotherapy compared with glimepiride in patients with type 2 diabetes. Diabetes Obes Metab 2011;13:348-356.

10. Ahren B, Johnson SL, Stewart M, et al.: HARMONY 3: 104-week randomized, double-blind, placebo- and activecontrolled trial assessing the efficacy and safety of albiglutide compared with placebo, sitagliptin, and glimepiride in patients with type 2 diabetes taking metformin. Diabetes Care 2014;37:2141-2148.
11. Klonoff DC, Buse JB, Nielsen LL, et al.: Exenatide effects on diabetes, obesity, cardiovascular risk factors and hepatic biomarkers in patients with type 2 diabetes treated for at least 3 years. Curr Med Res Opin 2008;24:275-286.

12. Diamant M, Van Gaal L, Guerci B, et al.: Exenatide once weekly versus insulin glargine for type 2 diabetes (DURATION-3): $3-$ year results of an open-label randomised trial. Lancet Diabetes Endocrinol 2014;2:464-473.

13. Trautmann M, Van Gaal L, Han J, et al.: Three-year efficacy and safety of exenatide once weekly: a pooled analysis of 3 trials. Diabetes 2015;64(Suppl. 1):A287.

14. MacConell L, Brown C, Gurney K, et al.: Safety and tolerability of exenatide twice daily in patients with type 2 diabetes: integrated analysis of 5594 patients from 19 placebo-controlled and comparator-controlled clinical trials. Diabetes Metab Syndr Obes 2012;5:29-41.

15. MacConell L, Gurney K, Malloy J, et al.: Safety and tolerability of exenatide once weekly in patients with type 2 diabetes: an integrated analysis of 4,328 patients. Diabetes Metab Syndr Obes 2015;8:241-253.

16. Sun F, Wu S, Guo S, et al.: Impact of GLP-1 receptor agonists on blood pressure, heart rate and hypertension among patients with type 2 diabetes: a systematic review and network meta-analysis. Diabetes Res Clin Pract 2015;110:26-37.

17. Starner CI, Schafer JA, Heaton AH, et al.: Rosiglitazone and pioglitazone utilization from January 2007 through May 2008 associated with five risk-warning events. J Manag Care Pharm 2008;14:523-531.

Address correspondence to: Robert R. Henry, MD Center for Metabolic Research VA San Diego Healthcare System 3350 La Jolla Village Drive (111G) San Diego, CA 92161

E-mail:rrhenry@outlook.com 